\title{
Spatial Distribution of Anopheles sinensis and Its Relationship with Natural Environmental Factors in Wanning County on Hainan Island, China
}

\author{
Liang Wen ${ }^{* * \#, ~ Q i n g h u a ~ L i 1 ~}{ }^{*}$, Minghe Lin ${ }^{2 *}$, Chengyi Li1, Chenyi Chu' ${ }^{1}$, Shaowen Weng ${ }^{2}$, \\ Wenyi Zhang ${ }^{1}$, Yong Wang ${ }^{1}$, Shenlong Li ${ }^{1}$ \\ ${ }^{1}$ Chinese PLA Center for Disease Control and Prevention, Beijing, China \\ ${ }^{2}$ Wanning Center for Disease Control and Prevention, Wanning County, Hainan Province, China \\ Email: "wenlty@sohu.com
}

How to cite this paper: Wen, L., Li, Q.H., Lin, M.H., Li, C.Y., Chu, C.Y., Weng, S.W., Zhang, W.Y., Wang, Y. and Li, S.L. (2021) Spatial Distribution of Anopheles sinensis and Its Relationship with Natural Environmental Factors in Wanning County on Hainan Island, China. Journal of Geographic Information System, 13, 36-51. https://doi.org/10.4236/igis.2021.131003

Received: January 4, 2021

Accepted: February 5, 2021

Published: February 8, 2021

Copyright $\odot 2021$ by author(s) and Scientific Research Publishing Inc. This work is licensed under the Creative Commons Attribution International License (CC BY 4.0).

http://creativecommons.org/licenses/by/4.0/

\begin{abstract}
Anopheles sinensis is widely distributed in Wanning County, it is necessary to understand the spatial distribution characteristics of Anopheles sinensis in order to maintain the elimination of malaria in Wanning. During May and October 2009, we sampled adult mosquitoes at 36 villages within Wanning County on Hainan island, and collected meteorological and geographical data at each sampling site. We used these data to analyze the spatial distribution of adult Anopheles sinensis mosquitoes, and logistic regression analysis was applied to explore the association of the spatial distribution of Anopheles sinensis with the geographical and meteorological factors. We found that the density of Anopheles sinensis showed a significant positive spatial correlation. From May to October, on the whole, the high-density area was located in the central part of Wanning County. But each month there was a relatively high-density cluster, and their location and range were not exactly the same. From east to west, the density of Anopheles sinensis increased initially and then decreased, but from south to north, there were different trends in the periods of May to August and September to October. Logistic regression analysis showed that the main environmental factors related with the distribution of Anopheles sinensis were land use type, soil type, distance to road, air pressure and relative humidity. These analysis results showed that the distribution of Anopheles sinensis had obvious spatial heterogeneity in Wanning County, which was related with geographical and meteorological factors. The mechanism of these environmental factors on the distribution of Anopheles sinensis needs to be further studied.
\end{abstract}

*These authors contribute equal to this work. 


\section{Keywords}

Anopheles sinensis, Spatial Distribution, Space-Time Clustering, Environmental Factors, Spatial Epidemiological Analysis

\section{Introduction}

Hainan Island, located in the South China Sea and separated from Leizhou Peninsula by the Qiongzhou Strait, makes up the majority of the Hainan Province, China. It was once one of the worst malaria endemic areas in China. In 2003, Hainan Province started implementing the Global Fund Malaria Project, which greatly contributed to the implementation of Hainan's malaria control program. Since 2010, Hainan had carried out malaria elimination according to the Malaria Elimination Action Plan (2010-2020), the number of malaria cases continued to decline, and realized "No local malaria cases reported" in 2012 [1]. On December 31, 2019, it officially confirmed that Hainan Province had reached the Malaria elimination target.

Anopheles mosquito is the sole vector of malaria. There are 37 species of Anopheles mosquito on Hainan Island. Exophagic, anthropophilic An. dirus and endophilic, anthropophilic An. minimus were the principal vectors in the period of high malaria prevalence in Hainan Island. An. sinensis, An. jeyporiensis candidiensis and several other Anopheles were secondary vectors [2]. With the development of society and economy, large areas of forests have been cut down to grow tropical cash crops such as rubber, areca nut and fruit, destroying breeding habitats for wild inhabiting mosquito species, and with long-term targeted mosquito control measures, the distribution and abundance of An. dirus and An. minimus have been drastically decreased [3]. An. sinensis is a type of exophagic and endophilic mosquito that feeds on both human and livestock blood. It is not an efficient malaria vector compared with An. dirus and An. minimus, but it is widely distributed on Hainan Island and has a large species population. With the development of agricultural mechanization, the drastic reduction of farm cattle has relatively increased the chances of the An. Sinensis mosquito, which used to feed on cattle blood, feeding on human blood, An. sinensis may become the mosquito species responsible for a low prevalence of malaria in Hainan Island, so it is necessary to monitor the spatial and temporal distribution of An. sinensis.

The dry season and the wet season are clearly different in Hainan, as the dry season (from November to April) is warm and dry while the wet season (from May to October) is hot and humid. The peak season of malaria overlapped with wet season during the malaria epidemic [4]. This study took Wanning County as the sample area, and analyzed the spatial distribution characteristics of adult $A n$. sinensis mosquitoes and their relationship with environmental factors in wet season, in order to formulate tailored mosquito control measures to maintain 
elimination of malaria in Hainan Island.

\section{Materials and Methods}

\subsection{Study Area}

Wanning County is located on the southeast coast of Hainan Island with a tropical monsoon climate featuring ample sunshine and precipitation. Wanning is mountainous in the west, hilly in the central and plain in the southeast. The three terrains account for $46 \%, 32 \%$ and $22 \%$ respectively, and the average elevation is 123 meters. The topography and geomorphology of Wanning is a good representation of that of Hainan Island.

Wanning used to be a malaria-endemic area. After joining the Malaria Programme of China Global Fund Round One in 2003, the incidence of malaria in Wanning began to decrease significantly. The elimination of malaria has been carried out comprehensively in Wanning since 2010 and reached the standard of elimination in 2017 [5]. The historical malaria hyper-endemic area of Wanning lies in the western and northwestern mountainous regions where An. minimus and $A n$. dirus were highly effective vectors. Although mosquito surveillance has shown that the population density of $A n$. sinensis is significantly higher than that of An. dirus and An. minimus at villages with high malaria incidence, $A n$. sinensis is not considered a major malaria vector and has not drawn the same attention as An. minimus and An. dirus during the high malaria transmission era [6].

\subsection{Mosquito Surveillance}

In this study, 36 adult mosquito monitoring sites were set up in 36 villages, 26 of which were located in the three historical malaria endemic towns (Nanqiao, Sangengluo and Beida) and 10 in non-malaria-endemic areas (Figure 1).

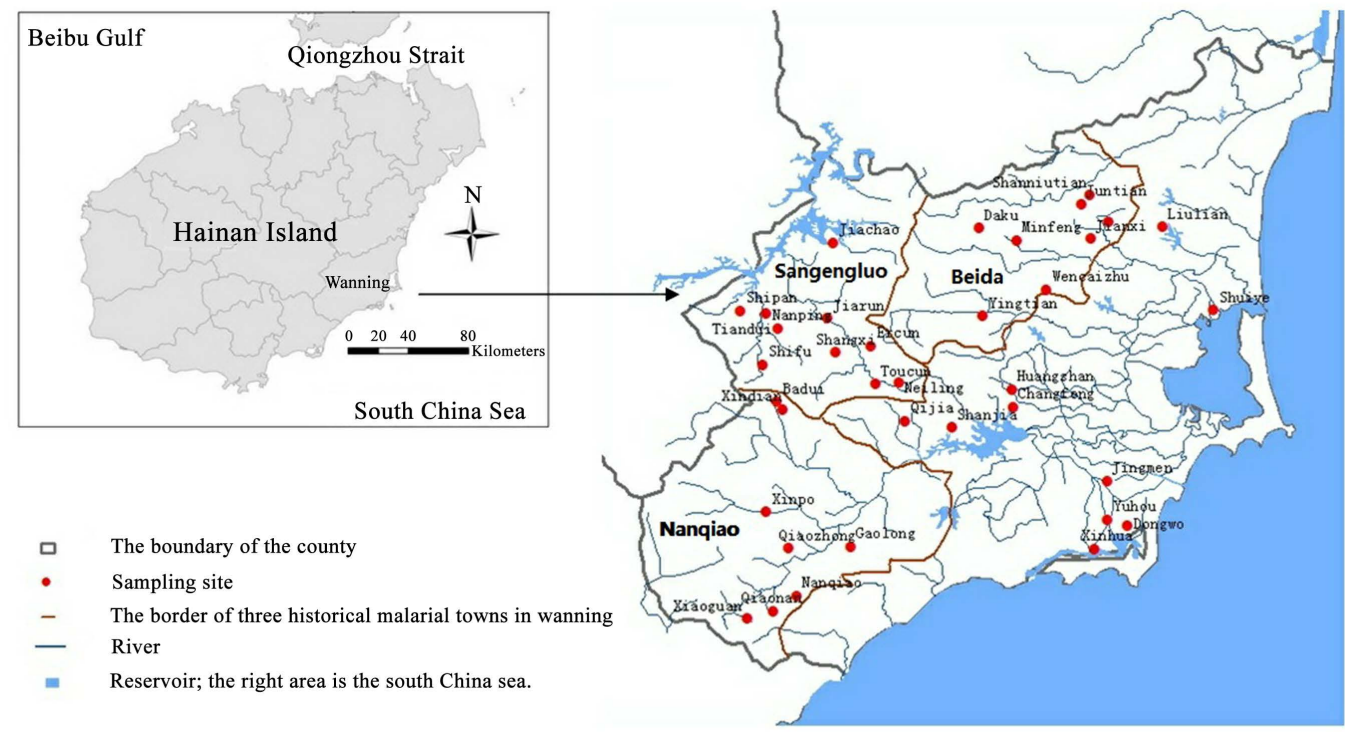

Figure 1. Study area and monitoring sites. 
At each monitoring site, adult mosquitoes were collected outdoors twice a month using human landing catch technique from May to October 2009, once in the middle of the month and once at the end of the month. Due to the time of peak activity of $A n$. sinensis is in the first half of the night [7] [8], mosquito sampling was carried out from 7 p.m. to 12 p.m. Informed consent was obtained from all the participants. Wanning Center for Disease Control and Prevention (CDC) provided medical supervision of participants. All collected mosquitoes were sent to the laboratory for classification. Densities of An. sinensis adult mosquito were measured as the number of An. sinensis adult mosquitoes captured per person-hour.

\subsection{Environmental Data Acquisition}

GPS receiver was used to record the geographical information (latitude, longitude and altitude) of each sampling site and the distance to the nearest household.

The distances from each sampling site to the nearest river and the nearest highway were calculated using spatial analysis in ArcGIS9.3 software (Environmental Systems Research Institute, Inc.).

The paper map of soil type distribution of Wanning County was obtained from Hainan Administration of Surveying, Mapping and Geoinformation. Soil type of each sampling site was extracted using spatial analysis in ArcGIS9.3 after the paper map being digitized.

The land use types of the sampling sites were classified into three categories: residential area, woodland and grassland respectively.

The temperature, air pressure and relative humidity of the sampling sites were recorded at the beginning of sampling using unified barothermohygrographs.

\subsection{Data Analysis}

Global Spatial autocorrelation analysis: Global spatial autocorrelation describes the overall distribution of observed objects in space to determine if there was spatial aggregation. Moran's I index was used to measures the spatial aggregation patterns and aggregation level of attribute variables across the study area. The Moran's I index was tested using standardized statistic Z values. Values of Moran's I index ranges from -1 to 1 , a positive value indicates tendency toward clustering while a negative value indicates tendency toward dispersion, a value of zero typically indicates no spatial autocorrelation, and the greater the absolute value of Moran's I is, the higher the degree of autocorrelation in spatial distribution is [9] [10]. In this study, the spatial autocorrelation tool in Arcgis10.2 was used to measure the value of Moran's I.

Space-time scanning cluster analysis: Scanning statistics are used to discover and evaluate the aggregation of events in pure time, pure space, or space-time dimensions by continuously moving scan window in time and/or space dimensions and recording the number of observations and expectations in the window for each position [11]. SaTScan9.4 software was used to carry out the purely spa- 
tial and spatio-temporal scanning cluster analysis of the density distribution of adult An. sinensis mosquitoes.

Because density was continuous data, the normal model was used to conduct the pure space clustering in order to find the high density aggregation area of An. sinensis in each month. For spatio-temporal analysis, the mosquito densities of each sampling site in each month were divided into 1, 2, 3, 4, and 5 levels from low to high, and ordinary model was used to conduct spatio-temporal clustering to clarify the spatial and temporal aggregation of An. sinensis in the whole surveillance period. The unit of space was defined by the coordinates of the sampling sites and the unit of time was one month. The maximum spatial cluster size was set to include up to $50 \%$ of the total sampling sites and the maximum size of the time frame was $50 \%$ of the surveillance period. For Monte-Carlo inference, 999 replications were performed.

Trend surface analysis: Trend surface analysis is a method based on low-order polynomials of spatial coordinates for estimating a regular grid of points from scattered observations. This approach is aimed to model the overall distribution of properties throughout space. It will sketch the global trend of distribution as a simplified surface. A trend surface model is a particular case of a bivariate regression model with two independent variables, the coordinates $\mathrm{X}$ and $\mathrm{Y}$ and a dependent variable, the thematic variable $\mathrm{Z}$ to be modeled [12] [13]. In this study, ArcGIS 10.2 software was used to analyze trend surface and draw trend analysis graph.

Logistic regression analysis: Bivariate logistic regression models were fitted to identify environmental variables associated with the presence of adult An. sinensis mosquitoes. Then, multivariate logistic regression model, incorporating variables significant at $P<0.05$ in the univariate analysis, was developed using a forward-stepwise procedure and assessed using the likelihood ratio test. All regression analyses were carried out with SPSS 20.0.

\section{Results}

\subsection{General}

Of the 36 sampling sites, four sites (Jiarun, Toucun, Shanniutian and Minfeng) were stopped sampling from July and one site (Qiaozhong) was stopped sampling in October due to lack of sampling personnel, all the five sites are located in the historical malaria high endemic region. Accumulated 1498 person-hours of sampling were carried out and a total of 619 An. sinensis mosquitoes were caught. The average density of An. sinensis captured in each month from May to October was $0.61,0.37,0.39,0.41$, and 0.29 per man-hour, respectively. The density in May was significantly higher than that in other months, and there was no significant difference in the density of other months (Friedman $\mathrm{M}$ test, $\mathrm{M}=$ 25.273, $P=0.000$ ). Of the 31 sampling sites that have completed all six months of surveillance, the highest average density from May to October was 1.08 per man-hour observed in Shanjia (see Figure 2). The highest density of single 


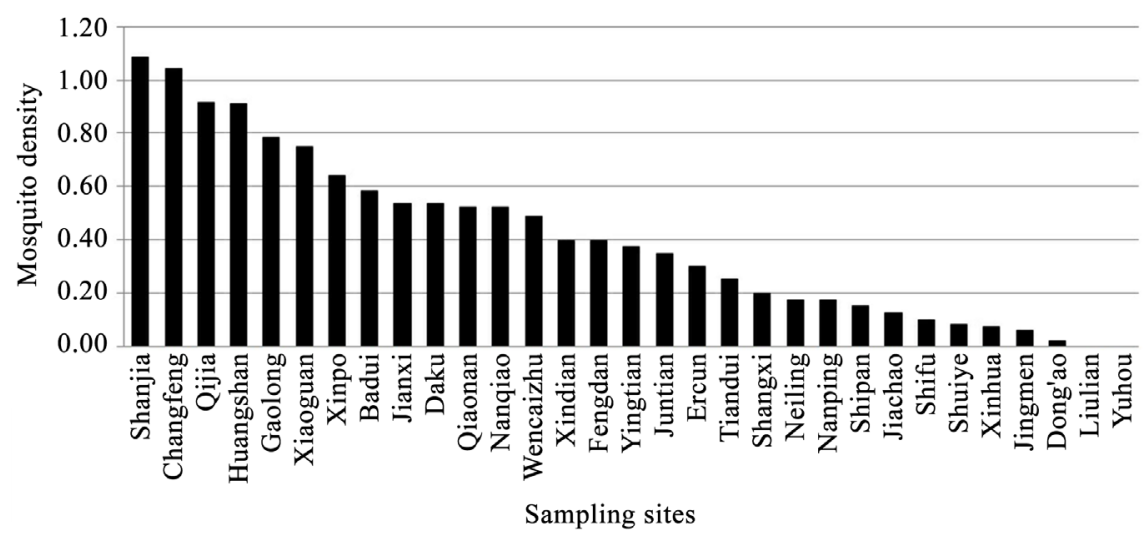

Figure 2. Average mosquito densities of 31 sampling sites during May and October, 2009 (unit: number of Anopheles sinensis captured per person-hour).

surveillance was 2.75 per man-hour observed in Shanjia in mid-May. No An. sinensis was captured during the whole surveillance period at two sampling sites, Yuhou and Liulian, which both lie in the historically low endemic area of malaria.

\subsection{Spatial Autocorrelation Analysis}

The global autocorrelation analysis showed that the distribution of An. sinensis in each month from May to October 2009 was spatial aggregation rather than random one. The highest level of aggregation of mosquito density was in October, the lowest level of aggregation was in June (Table 1).

\subsection{Spatial and Temporal Scanning Clustering}

Purely spatial scanning cluster analysis: It was shown that mosquito population was not distributed randomly in Wanning County according to analysis of purely spatial clustering of mosquito density from May to October 2009. With the maximum spatial cluster size of $50 \%$ of the total surveillance sites, the spatial clustering identified the most likely cluster with high An. sinensis mosquito density for each of the six months. The secondary cluster was only detected in September and was not statistically significant (Table 2). The cluster covered three sampling sites in May, two sites in June, sixteen sites in July, sixteen sites same as those of July in August, eight sites in September, and eight sites same as those of September in October (Figure 3). These results showed that the relatively high density clusters of each month were not fixed.

Spatio-temporal scanning cluster analysis: Spatio-temporal scanning cluster of An. Sinensis density in Wanning County from May to October, 2009 was tested by ordinary model. The most likely cluster with high mosquito density located in central area of Wanning County (Coordinates: 18.791N, 110.262E; radius: $5.56 \mathrm{~km}$; LLR: 17.636; $P=0.001$ ) where covered three sampling sites adjacent to Wanning Reservoir in non-malaria-endemic areas (Figure 4), and the time frame was from May to July. No secondary cluster was detected. 
Table 1. Global spatial autocorrelation of An. sinensis mosquito density in Wanning County from May to October, 2009.

\begin{tabular}{cccc}
\hline Month & Moran's I Index & Z-score & $P$-value \\
\hline May & 0.23 & 2.36 & 0.02 \\
Jun & 0.23 & 2.35 & 0.02 \\
Jul & 0.43 & 3.23 & 0.00 \\
Aug & 0.61 & 4.44 & 0.00 \\
Sept & 0.57 & 4.09 & 0.00 \\
Oct & 0.70 & 4.78 & 0.00
\end{tabular}

Table 2. Purely spatial An. sinensis clusters in Wanning County from May to October, 2009.

\begin{tabular}{cccccc}
\hline Month & Coordinates/radius & Mean inside & Mean outside & LLR $^{*}$ & $P$-value \\
\hline May & $(18.791 \mathrm{~N}, 110.262 \mathrm{E}) / 5.56 \mathrm{~km}$ & 1.75 & 0.46 & 12.47 & 0.01 \\
\hline Jun & $(18.791 \mathrm{~N}, 110.262 \mathrm{E}) / 4.03 \mathrm{~km}$ & 2.00 & 0.25 & 21.25 & 0.05 \\
\hline Jul & $(18.694 \mathrm{~N}, 110.180 \mathrm{E}) / 19.70 \mathrm{~km}$ & 0.64 & 0.16 & 8.85 & 0.03 \\
\hline Aug & $(18.694 \mathrm{~N}, 110.180 \mathrm{E}) / 19.70 \mathrm{~km}$ & 0.64 & 0.20 & 7.18 & 0.05 \\
\hline \multirow{2}{*}{ Sept } & $(18.903 \mathrm{~N}, 110.338 \mathrm{E}) / 10.91 \mathrm{~km}$ & 0.70 & 0.29 & 6.23 & 0.04 \\
\hline Oct & $(18.694 \mathrm{~N}, 110.180 \mathrm{E}) / 14.52 \mathrm{~km} *$ & 0.55 & 0.32 & 2.01 & 0.86 \\
\hline
\end{tabular}

${ }^{*}$ LLR: Log likelihood ratio, ${ }^{* *}$ secondary cluster.

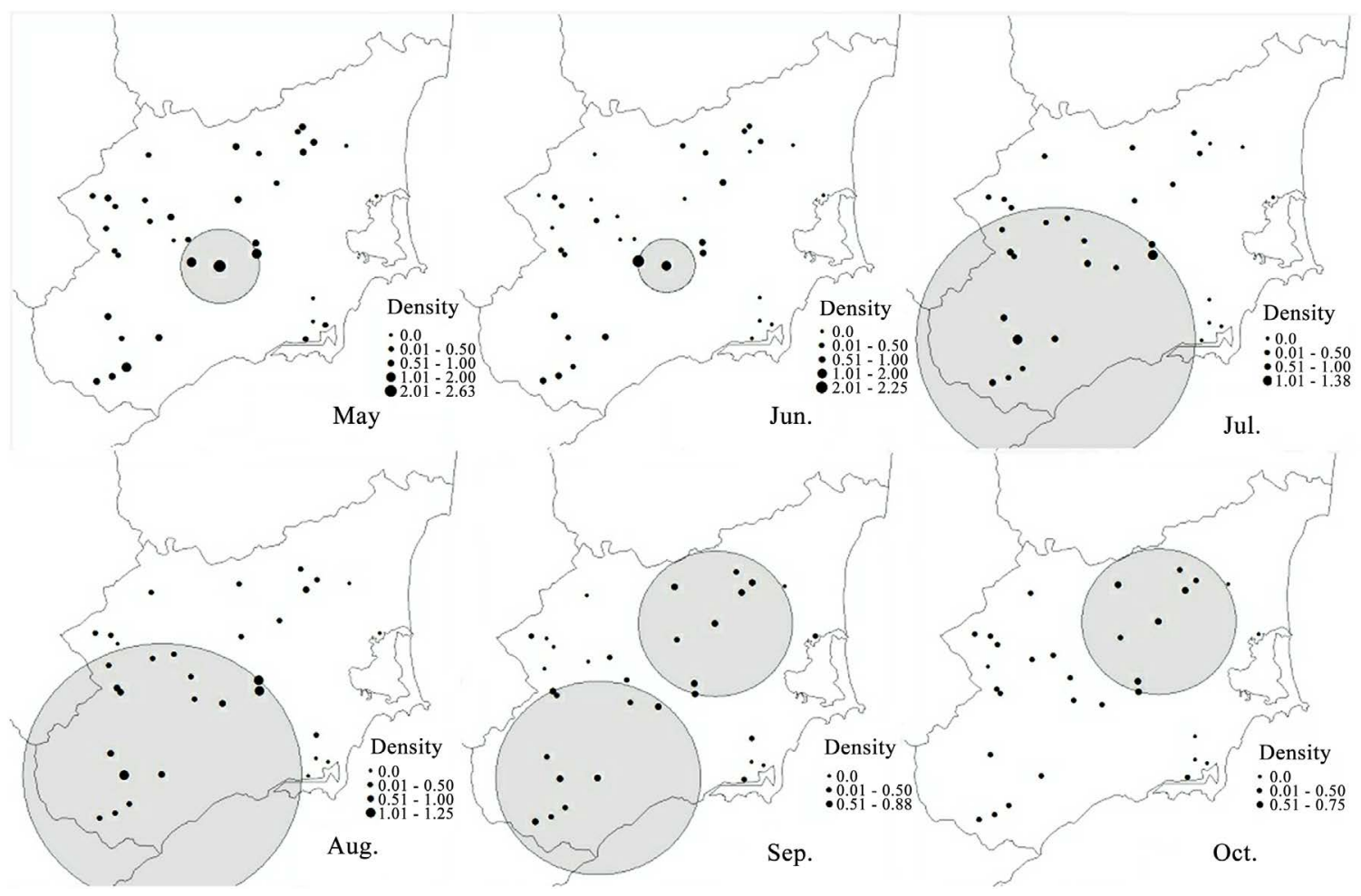

Figure 3. The monthly spatial clusters of An. sinensis in Wanning County from May to October, 2009. 


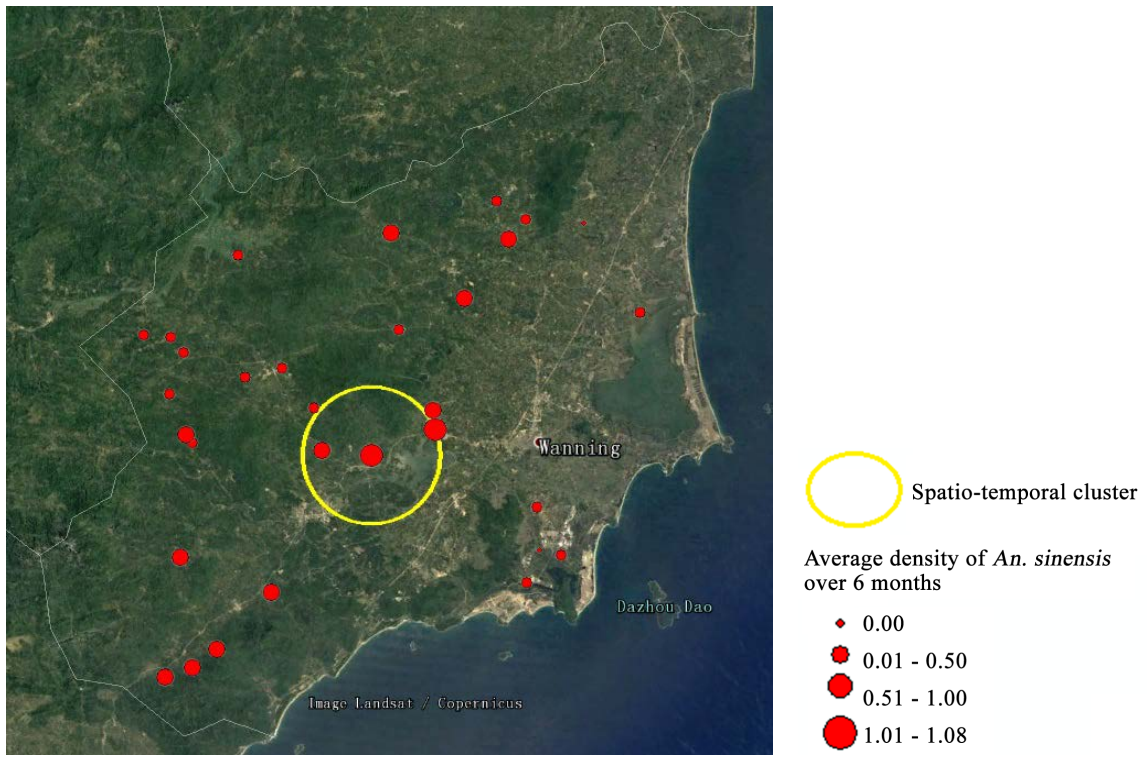

Figure 4. The location of the spatio-temporal cluster of An. sinensis mosquitoes in Wanning from May to October, 2009 (the map was taken from the Google earth. Imagery date: $12 / 31 / 2009)$.

\subsection{Trend Surface Analysis}

Trend surface analysis was employed to identify the geographic trends of the mosquito density. A coordinate system was created (one axis for each direction with $\mathrm{X}$ for West-East and Y for South-North) and projections of densities ( $\mathrm{Z}$ axis) reflected the variation trend of West-East and South-North orientation.

The result showed that density distribution of An. sinensis each month during May and October presented an obviously inversed " $U$ " shape from west to east, and presented a downward trend from May to August from south to north, a less obviously "U" shape from September to October (see Figure 5).

\subsection{Logistic Regression Modeling for Adult An. sinensis Mosquitoes Presence/Absence}

Univariate analysis: Univariate two-class logistic regression analysis was used to analyze the correlation between various environmental factors and whether or not An. sinensis was captured.

Univariate analysis was used to select environmental factors that were significantly associated with the presence of adult $A n$. sinensis mosquitoes. Bivariate logistic regression models combining presence of adult An. sinensis mosquitoes and various environmental characteristics of sampling site are presented in $\mathrm{Ta}$ ble 3. The results showed that the presence of adult An. sinensis mosquito was more likely in residential areas and sand shale yellow red soil areas, and was negatively correlated with distance to highway, distance to river, temperature, air pressure, and relative humidity, and was positively correlated with the distance to household. The presence of adult An. sinensis mosquito had no correlation with the sampling month or the elevation of sampling site. 


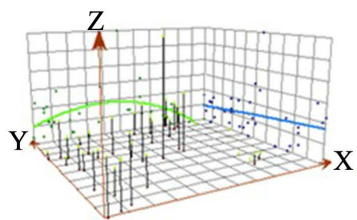

May

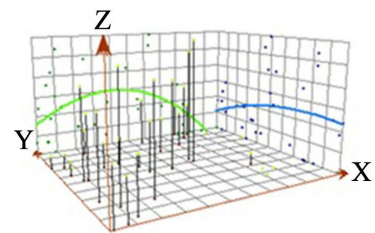

Aug.

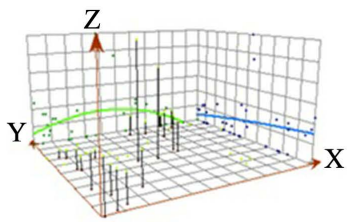

Jun.

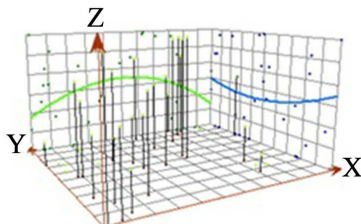

Sep.

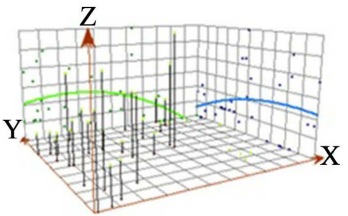

Jul.

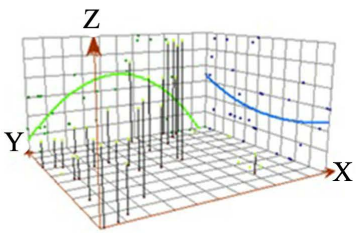

Oct.

$$
\text { X: longitude } \quad \text { Y: latitude } \quad \text { Z: density }
$$

Figure 5. Trend surface map of density of An. sinensis in Wanning County from May to October, 2009.

Table 3. Bivariate logistic regression results for presence of adult An. sinensis mosquitoes and the environmental factors in Wanning County.

\begin{tabular}{|c|c|c|c|c|c|}
\hline Environmental factor & B & S.E & Wals & Odd Ratio (95\% CI) & $P$-value \\
\hline Date & & & 10.07 & & 0.07 \\
\hline May & 0.73 & 0.42 & 3.04 & $2.065(0.91-4.66)$ & 0.08 \\
\hline Jun & -0.45 & 0.37 & 1.48 & $0.635(0.31-1.32)$ & 0.22 \\
\hline Jul & 0.21 & 0.40 & 0.27 & $1.228(0.57-2.66)$ & 0.60 \\
\hline Aug & 0.37 & 0.40 & 0.85 & $1.447(0.66-3.18)$ & 0.36 \\
\hline Sept & 0.05 & 0.39 & 0.02 & $1.053(0.49-2.26)$ & 0.90 \\
\hline Oct & - & - & - & 1.00 & - \\
\hline Land use type & & & 37.53 & & 0.00 \\
\hline Woodland & -1.82 & 0.30 & 37.35 & $0.16(0.09-0.29)$ & 0.00 \\
\hline Grassland & -1.36 & 0.34 & 15.82 & $0.26(0.13-0.50)$ & 0.00 \\
\hline Residential area & - & - & - & 1.00 & - \\
\hline Soil type & & & 53.77 & & 0.00 \\
\hline Neritic yellow laterite & 0.84 & 0.82 & 1.04 & $2.32(0.46-11.62)$ & 0.31 \\
\hline Granite yellow laterite & 2.61 & 0.80 & 10.54 & $13.62(2.82-65.91)$ & 0.00 \\
\hline Granite yellow red soil & 2.87 & 0.80 & 12.90 & $17.57(3.68-83.95)$ & 0.00 \\
\hline Sand shale yellow red soil & 4.91 & 1.28 & 14.70 & $135.00(11.00-1657.37)$ & 0.00 \\
\hline Paddy soil & - & - & - & 1.00 & - \\
\hline Distance to highway & -0.00 & 0.00 & 9.10 & $1.00(1.00-1.00)$ & 0.00 \\
\hline Distance to river & -0.02 & 0.00 & 25.91 & $0.98(0.98-0.99)$ & 0.00 \\
\hline Distance to household & 0.01 & 0.00 & 7.91 & $1.01(1.00-1.01)$ & 0.01 \\
\hline Elevation & 0.00 & 0.00 & 0.78 & $1.00(1.00-1.01)$ & 0.38 \\
\hline Temperature & -0.40 & 0.10 & 14.90 & $0.67(0.55-0.82)$ & 0.00 \\
\hline Air pressure & -0.18 & 0.04 & 26.11 & $0.84(0.78-0.90)$ & 0.00 \\
\hline Relative humidity & -0.09 & 0.02 & 15.50 & $0.91(0.87-0.95)$ & 0.00 \\
\hline
\end{tabular}


Multivariate analysis: In order to comprehensively evaluate the effect of environmental factors on the presence of adult An. sinensis population, multivariate logistic regression was used to develop minimum adequate models with all significant variables $(P<0.05)$ in Table 3. Stepwise logistic regression analysis showed that the five environmental factors (land use type, soil type, and distance to highway, air pressure and relative humidity) were the influencing factors of presence of adult $A n$. sinensis (Table 4). Land use type and soil type had strong correlation with the presence of An. sinensis, and distance to highway, air pressure and relative humidity were negative correlation with the presence of $A n$. sinensis. Three covariates, distance to river, distance to household and temperature, were excluded from the model. The $R^{2}$ of the regression equation was 0.489 , and using the predicted probability of 0.5 as a cut-off point for classification, the correct rate of classification was $80.3 \%$.

\section{Discussion}

In China, An. sinensis is the main or sole malaria vector in the region north of $34^{\circ}$ north latitude. It is an important malaria vector in the plains, especially the main vector for Plasmodium vivax in rice growing areas [14]. Hainan island lies south of $21^{\circ}$ north latitude, the main vectors of malaria during the high-malaria transmission era were An. dirus. and An. minimus. They have a higher susceptibility to Plasmodium vivax and Plasmodium falciparum than An. Sinensis [15] [16]. The spatial and temporal distribution characteristics of An. sinensis on Hainan Island have been rarely studied since the beginning of this century. However, due to long-term targeted mosquito control and environmental changes caused by socioeconomic development, the mosquito populations of An. dirus and An. minimus have been greatly reduced in recent years [17].

Table 4. Stepwise logistic regression results for presence of adult An. sinensis mosquitoes and the environmental variables in Wanning County.

\begin{tabular}{cccccc}
\hline Environmental factor & B & S.E & Wals & Odd Ratio (95\% CI) & $P$-value \\
\hline Land use type & & & 47.15 & & 0.00 \\
Woodland & -3.39 & 0.51 & 44.52 & $0.03(0.01-0.09)$ & 0.00 \\
Grassland & -2.49 & 0.49 & 25.71 & $0.08(0.03-0.22)$ & 0.00 \\
Residential area & - & - & - & 1.00 & - \\
\hline Soil type & & & 18.39 & & 0.01 \\
Neritic yellow laterite & 2.61 & 1.02 & 6.60 & $13.65(1.86-100.21)$ & 0.01 \\
Granite yellow laterite & 3.20 & 0.92 & 12.18 & $24.40(4.06-146.74)$ & 0.00 \\
Granite yellow red soil & 3.38 & 0.93 & 13.12 & $29.26(4.71-181.83)$ & 0.00 \\
Sand shale yellow red soil & 5.28 & 1.43 & 13.71 & $196.76(12.01-3224.32)$ & 0.00 \\
$\quad$ Paddy soil & - & - & - & 1.00 & - \\
\hline Distance to highway & -0.01 & 0.00 & 10.94 & $0.99(0.99-1.00)$ & 0.00 \\
Air pressure & -0.10 & 0.04 & 5.92 & $0.91(0.84-0.98)$ & 0.02 \\
Relative humidity & -0.13 & 0.04 & 11.47 & $0.877(0.81-0.95)$ & 0.00 \\
\hline
\end{tabular}


During the surveillance period of this study, only three An. dirus mosquitoes and no An. minimus mosquito were captured. An. sinensis has a significant population advantage over An. dirus and An. minimus in the population structure of malaria vector Anopheles mosquitoes on Hainan Island, the population distribution characteristics and malaria transmission efficiency of the An. sinensis need further attention in order to eliminate malaria on Hainan island.

Anopheles sinensis has a broad adaptability to the breeding environment. The main breeding place is the paddy field and its irrigation systems. The seasonal variation of the population size is closely related to climate change and farming system in different areas. In China, adult $A n$. sinensis are active throughout the year in regions south of $27^{\circ}$ north latitude, survive the winter in semi-hibernation in areas between $28^{\circ}$ and $33^{\circ}$ north latitude, and enter hibernation in winter in regions north of $33^{\circ}$ north latitude [18]. This survey shows that An. sinensis are active throughout the wet season from May to October in Wanning County, and the density in May is higher than that in the other months. This is consistent with a survey conducted in several districts in Hainan island in 2012, in which the peak of adult mosquito density appeared from April to June [19], but is inconsistent with the observations obtained in China mainland which was from July to August [20]. Perhaps, the reason is that the temperature and humidity conditions in Hainan Island were suitable for breeding and developing of mosquitoes all year round, however, with the increase of precipitation and tropical low-pressure cyclone from July to October each year, mosquito breeding sites and habitats would be destroyed, resulting in a certain drop in mosquito density.

The analysis result showed that the density of adult An. sinensis had significant positive spatial correlation. In other words, mosquito densities of neighboring monitoring sites were closer, indicating the distribution of adult An. sinensis was dependent on the spatial location. Changes of the intensities of correlation in six months suggest that seasonal changes of some environmental factors affecting the density of adult An. sinensis may have an impact on the intensity of this dependence. Positive spatial correlation will inevitably result in spatial aggregation. The monthly space scanning statistics showed that there was a relative high density spatial aggregation area in each month, the changes of location and extent of monthly aggregation areas suggested that the intensity of mosquito density changes of different sampling sites were not consistent in different seasons. The space-time scanning from May to October showed that during the whole surveillance period, the high density aggregation area of adult $A n$. sinensis was located in the northwest of Wanning reservoir and the time frame was from May to July. This area is not the traditional malaria high-endemic area in Wanning and is obviously inconsistent with the spatial distribution of malaria cases in Wanning County in 2009 [21]. Whether this can suggest that An. sinensis is not the main vector of malaria in Wanning County needs more in-depth research, because in addition to mosquito vectors, there are many other factors that affect the malaria epidemic such as the presence of malaria case and wheth- 
er and how prevention and control measures are taken.

Besides the characteristics of spatial distribution of adult An. sinensis, this study explored the key natural environmental factors related with this distribution feature. Logistic regression analyses showed that land use type, soil type, distance to highway, air pressure, and relative humidity were major factors related with the presence of adult An. sinensis.

Adult $A n$. sinensis was more likely to be found in residential areas than in woodland and grassland, as they are easier to find vertebrate host to suck blood in residential areas. Soil is closely related to insects, it not only indirectly affects insects as the plants grow, but also is places where insects live. Studies shows that the duration of immature life stages of the mosquitoes is inversely related to soil-substrate organic matter content and soil moisture has an impact on the density of mosquitoes in the area [22] [23]. Shililu reported that the absolute moisture content of the soil is not the only factor that determines the successful rate of hatching anopheline eggs, rather, soil moisture, together with the rate of drying, physiological factors associated with the age of the egg, and the type of soil in which the egg rests, likely influences survival [24]. The mechanism how soil type affects distribution of Anopheles mosquitoes in Wanning needs further investigation. This study shows that the closer the monitoring point is to the highway, the greater the possibility of presence of adult An. sinensis, this may be due to some unknown indirect correlations that require further analysis.

Meteorological factors directly affect the mosquito density, because they affect not only reproduction and survival of mosquitoes but also their activities. According to Ma Aimin's research, total annual precipitation, mean annual air pressure, precipitation of the wettest quarter, and minimum temperature of the coldest month strongly influenced the distribution of An. sinensis [25]. The influence of temperature on mosquito populations is highly complex, as the effects of temperature on various processes are non-linear and difficult to predict. Some surveys have shown a positive correlation between mosquito density and temperature [26] [27] [28]. However, some other surveys showed a negative correlation as we observed in Wanning [29]. The relationship between air pressure and mosquito density also has different conclusions, these different conclusions may be due to differences in mosquito species and basic climatic conditions. For example, a survey conducted in the central Arctic showed that as air pressure rose, the probability of mosquito presence increased and the relationship between air pressure and activity level was also positive [30], while this study shows that the air pressure is inversely related to the presence of $A n$. sinensis and consistent with the findings of several surveys conducted in China which have shown that there is a significant negative correlation between air pressure and mosquito density, and the variation trend of mosquito density can be predicted by using the air pressure fitting model [28] [31] [32]. Hainan Island has high relative humidity, the relative humidity recorded in this study ranged from $68 \%$ to $97 \%$ with an average of $80 \%$. This survey shows that the relative humidity is inversely related to the presence of $A n$. sinensis, which is consistent with Ferreira's survey 
result that humidity above $75 \%$ had a negative effect on mosquito abundance [33].

Based on the close relationship between malaria and Anopheles mosquitoes and the high prevalence seasons of malaria in Hainan Island, this study carried out adult Anopheline mosquitos' surveillance in Wanning County from May to October, 2009, and analyzed the spatial distribution characteristics and environmental factors of An. sinensis population. The results showed that the spatial distribution of the high density areas of adult An. sinensis was inconsistent with the spatial distribution of the high incidence areas of malaria in Wanning. The high prevalence area of malaria has been located in the mountainous forest area of southwest Wanning for many years [21], however, the high density area of An. sinensis in this survey was located in the central hilly area. The density of An. sinensis in May was significantly higher than that in the other months, but the peak of malaria epidemic in Wanning was usually in summer and autumn. Whether this indicates that An. sinensis is not the main vector for maintaining the low level of malaria prevalence in Wanning County needs further study because many socio-economic factors also affect the malaria epidemic in addition to mosquito vectors.

\section{Conclusion}

The spatial distribution of An. sinensis adult mosquitoes in Wanning shows strong spatial heterogeneity, which is related to geographical and meteorological factors. Using the method of spatial epidemiological analysis, the spatial distribution characteristics of $A n$. sinensis mosquitoes and their influencing factors could be intensively explored to provide a basis for the local anti-mosquito strategies. Hainan Island is adjacent to Southeast Asia where malaria is widespread. As China builds Hainan Island into an international tourism island, population movement between Hainan Island and neighboring countries will be more frequent. This increases the risk of resurgence of malaria in Hainan Island, and monitoring and controlling mosquito vectors remains an important task of maintaining elimination of malaria in Hainan Island. To clarify the ecological habits of An. sinensis in Hainan Island for accurately forecasting the spatial and temporal distribution of An. sinensis, further comprehensive surveys are needed.

\section{Acknowledgements}

Special thanks to Chen X, Lin F, Fu JX, Hu MG, Tao Zh, Zhong ShCh, Zhong $\mathrm{XH}, \mathrm{Xu}$ ChH, Lin DX, Zong ZhH, Liu WX, Zhou ChAn, Lin L, Huang DY, Ji XiY, Li R, Huang G, Tang YP, Li PSh, Shi CB, Huang DY, Lu JH, Huang GP, Chen HSh, Shi LB, Lin XL, Huang HW, Huang HR, Xu ZhW, Wan CQ, Huang ShJ, Huang ZhL, Huang ZhX, Wang DS, Qiu XH, Chen QH, Wang Y, Jiang HY, Wen XZh, Zhang YP, Lan TT, Cui DY, Lin SQ, Chen B, Lu LL, and Wu Sh for their toilsome field mosquitoes sampling.

This study was supported by the National Natural Science Foundation of China (No. 30872151). 


\section{Conflicts of Interest}

The authors declare no conflicts of interest regarding the publication of this paper.

\section{References}

[1] Sun, D.W., Li, Y.C., Wang, G.Z. and Wang, S.Q. (2016) Mid-Term Assessment Report of Malaria Elimination Action Plan in Hainan. China Tropical Medicine, 16, 338-342.

[2] Sun, D.W., Wang, S.Q., Zeng, L.H., Li, S.G. and Zhuo, K.R. (2014) Survey of the Diversity of Anopheles Species in Hainan Province. Journal of Pathogen Biology, 9 , 271-274.

[3] Zeng, L.H., Wang, S.Q., Liu, Y., Zhao, W., Li, S.G., He, C.H., et al. (2015) Analysis of the Surveillance Data about Malaria Vector in Hainan from 2005 to 2014. China Tropical Medicine, 15, 1436-1440.

[4] Zeng, X.L. (2015) Study on the Distribution Characteristics of Malaria Epidemic Situation and Its Environmental Factors in Hainan Province. Ph.D. Thesis, School of Military Preventive Medicine, Third Military Medical University, China.

[5] Lin, M.H., Wang, S.Q., Wen, L., Zhu, D.C., Huang, S.L., Tao, Z., et al. (2018)Course of Malaria Control in Half a Century and Its Elimination in Wanning, Hainan. China Tropical Medicine, 18, 324-329.

[6] Zeng, L.H., Wang, Z.G., Guo, R.N. and Lan, X.H. (2000) Survey of Anopheles Mosquitoes at Malaria Outbreak Sites and High Malaria Prevalence Villages in Hainan Province. Hainan Medical Journal, 11, 3-4.

[7] Li, J.H., Qin, Y.X., Du, J.F. and Lin, Y.J. (2006) Population Composition and Activity Rhythm of Anopheles Mosquitoes in Wangdian, Guangxi Province, China. Journal of Applied Preventive Medicine, 12, 345-347.

[8] Zhou, H.N., Zhang, Z.X., Li, C.F., Wu, C., Wang, P.Y. Chris, C., et al. (2005)The Role of the Malaria Vectors in the Upper Valley of Mekong River in Yunnan. Chinese Journal of Parasitic Diseases Control, 18, 407-411.

[9] Zhou, X.N. (2009) Spatial Epidemiology. Science Press, Beijing.

[10] Jiang, Q.W. and Zhao, F. (2009) Application of Spatial Autocorrelation Method in Epidemiology. Chinese Journal of Epidemiology, 32, 539-546.

[11] Kulldorff, M. (2015) SaTScan ${ }^{\mathrm{TM}}$ User Guide for Version 9.4. https://www.satscan.org/

[12] Wen, X.Y., Peng ,B., Hu, S., Wen, W., Chen, J.P. and Que, P. (2015) Spatial Analysis of Birth Defects from 1996 to 2012 in China Based on Geographic Information System. Journal of Shanghai Jiaotong University (Medical Science), 35, 107-111.

[13] Tang, G.A. and Yang, X. (2006) ArcGIS Spatial Analysis Experiment Tutorial. Science Press, Beijing.

[14] Liu, Q.Y. and Liu, X.B. (2010) Prevention and Control of Vector Anopheles: A Key Approach for Malaria Elimination in China. Chinese Journal of Vector Biology and Control, 21, 409-413.

[15] Li, Z.H. (1998) The Experiment Report of the Sensitivity of An. minimus, An. dirus and An. sinensis to Plasmodium vivax and Plasmodium falciparum in Simao Prefecture. Journal of Medical Pest Control, 14, 11-12.

[16] He, D.X., Xu, Z.G., Ye, Y.Y. and Long, Z.P. (1983) Experimental Study on the Suscep- 
tibility of Anopheles minimus and Anopheles dirus to Plasmodium vivax and Plasmodium falciparum. Chinese Journal of Parasitology and Parasitic Diseases, 1, 83-87.

[17] Chen, W.J., Wu, K.C., Lin, M.H. and Li, C.X. (2007) Great Achievements of Anti-Malaria for a Half Century and the Present Technical Problems in Hainan Island. China Tropical Medicine, 7, 2013-2016.

[18] Chinese Ministry of Health, Department of Disease Control and Prevention (2007) Malaria Prevention and Treatment Handbook. 3rd Edition, People's Medical Publishing House, Beijing.

[19] He, C.H., Zhao, W., Wang, S.Q., Zeng, L.H., Li, S.G. and Ou, T.T. (2014) Analysis of Mosquito Density and Seasonality in Urban Areas of Hainan Province, China in 2012. Chinese Journal of Vector Biology and Control, 25, 15-17.

[20] Wan, X.N. (2012) Ecology Investigation of Anopheles Sinensis at Yellow River Delta. Journal of Medical Pest Control, 28, 794-795.

[21] Wen, L., Li, C.Y., Lin, M.H., Yuan, Z.Q., Huo, D.H., Li, S.L., et al. (2011) Spatio-Temporal Analysis of Malaria Incidence at the Village Level in a Malaria-Endemic Area in Hainan, China. Malaria Journal, 10, Article No. 88. https://doi.org/10.1186/1475-2875-10-88

[22] Olayemi, I.K. and Ojo, V.O. (2013) Immature Development of the Malaria Vector Mosquito, Anopheles gambiae S.L. (Diptera: Culicidae), in Relation to Soil-Substrate Organic Matter Content of Larval Habitats in Northcentral Nigeria. Pakistan Journal of Biological Sciences, 16, 135-140. https://dx.doi.org/10.3923/pjbs.2013.135.140

[23] Shaman, J., Stieglitz, M., Stark, C., Le, B.S. and Cane, M. (2002) Using a Dynamic Hydrology Model to Predict Mosquito Abundances in Flood and Swamp Water. Emerging Infectious Diseases, 8, 6-13.

[24] Shililu, J.I., Grueber, W.B., Mbogo, C.M., Githure, J.I., Riddiford, L.M. and Beier, J.C. (2004) Development and Survival of Anopheles gambiae Eggs in Drying Soil: Influence of the Rate of Drying, Egg Age, and Soil Type. Journal of the American Mosquito Control Association, 20, 243-247.

[25] Ma, A.M., Wang, J.F., Wang, D.Q. and Ren, Z.P. (2014) Prediction of Potential Distribution of Anopheles sinensis in China Based on MaxEnt. Chinese Journal of Vector Biology and Control, 25, 393-398.

[26] Jiang, Z., He, J.H., Xiao, L., et al. (2011) Monitoring the Mosquito Density, Rainfall and Temperature of Chengdu during 2006 to 2010. Journal of Medical Pest Control, 27, 817-818.

[27] Wang, J.N., Ling, F., Guo, S., Hou, J. and Gong, Z.Y. (2015) Study on the Relevant Meterological Factors Influencing Mosquito Density in Zhejiang Province. Chinese Journal of Vector Biology and Control, 26, 464-466, 470.

[28] Dai, P.F., Zhao, J.Y., Liu, M.D., Kong, X.S., Zhai, R.F., Wang, H.J., et al. (2011) Analysis on the Influence of Meteorological Parameters on Mosquito Density. Chinese Journal of Vector Biology and Control, 22, 547-549.

[29] Zhong, J. and He, L.H. (2015) Advances in Research on Impacts of Meteorological Factors on Mosquito Density. Chinese Journal of Vector Biology and Control, 26, 95-99.

[30] Witter, L.A., Johnson, C.J., Croft, B., Gunn, A. and Poirier, L.M. (2012) Gauging Climate Change Effects at Local Scales: Weather-Based Indices to Monitor Insect Harassment in Caribou. Ecological Application, 22, 1838-1851. https://doi.org/10.1890/11-0569.1

[31] Liu, F.R., Han, N., Zhang, Q.W., Dong, J. and Liang, X.S. (2010) Analysis on Mosquito Monitoring and Its Influential Factors in Longgang District of Shenzhen from 
2008 to 2009. Practical Preventive Medicine, 17, 2393-2396.

[32] Zhang, S.J., Zhao, X., Tang, C., Yang, B.H., Ma, T.Z., Li, C.Q., et al. (2015) Study on Meteorological Factors to Mosquito Density Surrounding Capital Airport. Chinese Journal of Public Health Management, 31, 10-11, 14.

[33] da Cruz Ferreira, D.A., Degener, C.M., de Almeida Marques-Toledo, C., Bendati, M.M., Fetzer, L.O., Teixeira, C.P., et al. (2017) Meteorological Variables and Mosquito Monitoring Are Good Predictors for Infestation Trends of Aedes aegypti, the Vector of Dengue, Chikungunya and Zika. Parasites \& Vectors, 10, Article No. 78. https://doi.org/10.1186/s13071-017-2025-8 